\title{
Statistics of inter-particle distances and angles in plasmas
}

\author{
D.V. Fisher ${ }^{\mathrm{a}}$ and Y. Maron \\ Faculty of Physics, Weizmann Institute of Science, Rehovot 76100, Israel
}

Received 27 October 2000 and Received in final form 30 January 2001

\begin{abstract}
Accurate treatment of the plasma density effects requires a detailed knowledge of the spatial distribution of individual ions around a test ion. In the present work, rigorous expressions are derived for the main 2- and 3-particle spatial distribution functions involving the nearest neighbor (NN) and the nextnearest neighbor (NNN) ions. These expressions, valid for both ideal and nonideal plasmas, present the distributions as functionals of the potentials $U_{\mathrm{NN}}$ and $U_{\mathrm{NNN}}$ at the nearest and next-nearest ion locations. All of the distribution functions except one are derived and discussed in the present work for the first time ever. For utilization of our results in practical calculations, we suggest semi-empirical expressions for $U_{\mathrm{NN}}$ and $U_{\mathrm{NNN}}$ in the ion-ion coupling parameter range $0 \leq \Gamma<1$. In order to test the accuracy of our expressions for $U_{\mathrm{NN}}$ and $U_{\mathrm{NNN}}$ we conduct Molecular Dynamics (MD) simulations. The simulations utilize the pure Coulomb particle-particle interaction potentials, regularized at close range to avoid classical Coulomb collapse, and are free from the assumptions made to find $U_{\mathrm{NN}}$ and $U_{\mathrm{NNN}}$. Thus, the results of the MD simulations provide an independent test of our theoretical results. Excellent agreement has been found between the results of the theory and of the MD simulations. Finally, we outline the implications of the present findings on the problem of tunneling and charge exchange in dense plasmas.
\end{abstract}

PACS. 52.25.-b Plasma properties - 34.10.+x General theories and models of atomic and molecular collisions and interactions (including statistic theories, transition state, stochastic and trajectory models, etc.) - 52.65.Cc Particle orbit and trajectory

\section{Introduction}

Progress in dense plasma physics necessitates a detailed account of the effects of the plasma environment on ionic bound states. In plasmas, a potential that binds an atomic (ionic) electron to its parent ion is distorted by the potentials of the surrounding ions and free electrons. In dilute plasmas, the mean interionic distance is much larger than the average radii of the bound states of sufficiently low principal quantum number. Therefore, in dilute plasmas, the total electric field of the surrounding particles does not vary significantly over the span of the wavefunction, and the Uniform Local Microfield (ULM) approximation is adequate [1] for describing the effect of the plasma environment [2] on those states. In dense plasmas, however, the mean inter-ionic distance is small, thus the applicability of the ULM approximation is restricted to the very low bound states, or in extreme cases to none at all. A correct treatment of higher-lying quantum states requires a more accurate description of the binding potential, i.e., necessitates an account of the spatial distribution of individual neighbor ions around the test ion. This is of particular importance for the treatment of transient quasimolecular states in dense plasmas [3-5] and for the study of the charge exchange [6] in general. In these cases the elec-

\footnotetext{
a e-mail: fndima@plasma-gate.weizmann.ac.il
}

tron quantum states that span inter-ionic distances must be considered. For these states the ULM approximation obviously fails. The problem of determining the effective statistical weights of bound states $[7-10]$ in dense plasmas has previously been treated primarily in the ULM approximation $[8,9]$. However, in this case, too, the wavefunctions of the states in question span inter-ionic distances, and the use of the detailed knowledge on the spatial distribution of the neighbor ions can improve the precision significantly. An accurate method for the determination of the effective statistical weights of the bound states based on the present findings will be reported separately [11]. The use of the accurate expressions for the effective statistical weights will allow the plasma equation-of-state and Rosseland opacity calculations (see, e.g., [12]) to attain higher precision [13]. Finally, being of basic interest as a part of statistical physics, the study of spatial distributions of the neighbor ions will also improve the general understanding of plasma density effects on transition rate coefficients and spectral line profiles $[1,10,14-16]$. In the treatments published so far the NN ion spatial distribution was obtained by Monte-Carlo $[4,17,18]$ or MD [16] simulations. However, the use of computer simulation routines to determine the NN distribution for each type of ions for each set of local thermodynamic conditions in inhomogeneous transient plasmas is not feasible. Analytical expressions, therefore, must be found for the spatial distribution of the 
$\mathrm{NN}$ ion and for the spatial and angular distributions of a few (at least one) next-nearest ions.

In this paper we report the results of both theoretical studies and quasiclassical MD simulations of the spatial distributions of ions around a test ion in nonideal plasmas. We introduce the probability $P_{0}(r)$ to find no ions (of a given type) within $r$ from the test ion, the distribution function $P_{\mathrm{NN}}(r)$ of the distance $r$ between the test ion and its NN ion, the distribution $P_{\mathrm{NNN}}(\rho)$ of the distance $\rho$ between the test ion and its NNN ion, the simultaneous distribution $P_{\mathrm{NNN}}(r, \rho)$ of the distances $r$ and $\rho$, and the distribution function $P_{\text {rat }}(\zeta)$ for the ratio $\zeta=\rho / r$. To the best of our knowledge, in the present work for the first time ever the distribution functions $P_{0}(r), P_{\mathrm{NNN}}(r, \rho)$, $P_{\mathrm{NNN}}(\rho)$, and $P_{\text {rat }}(\zeta)$ are studied and the expressions for them are given. As yet, only an expression for $P_{\mathrm{NN}}(r)$ has been published [10]. In Section 2 we describe our theoretical model and derive the exact expressions for the distribution functions $P_{0}(r)$ and $P_{\mathrm{NN}}(r)$ as the functionals of the potential $U_{\mathrm{NN}}(r)$ at the NN ion location. Next, we suggest and discuss an analytical expression for $U_{\mathrm{NN}}(r)$ valid for the ion-ion coupling parameter range $0 \leq \Gamma<1$. This provides for $P_{0}(r)$ and $P_{\mathrm{NN}}(r)$ the closed expressions that become exact in the weak coupling limit. In Section 3 we present both strict expressions and approximate analytical expressions for the distribution functions $P_{\mathrm{NNN}}(\rho)$ and $P_{\mathrm{NNN}}(r, \rho)$. In the study of non-resonant charge exchange $[6,19]$ and of the field ionization due to the NN ion effect (originally in [20]), the tunneling is assumed to take place in the field of the $\mathrm{NN}$ ion alone. To take into account the effect of the other surrounding ions, and foremost of the NNN ion, one needs to know the distribution function $P_{\text {rat }}(\zeta)$ of the distance ratio $\zeta$. The expressions for this distribution are also presented in Section 3. In order to test independently our theoretical results, we conducted the quasiclassical MD simulations for $P_{\mathrm{NN}}(r), P_{0}(r), P_{\mathrm{NNN}}(\rho)$, and $P_{\text {rat }}(\zeta)$. The simulation results are presented in Section 4. We also simulate and discuss the distribution function $P(\theta)$ of the angle $\theta$ between the directions from the test ion to the $\mathrm{NN}$ ion and from the test ion to the NNN ion. The discussion of the assumptions made in order to obtain the approximate analytical expression for $U_{\mathrm{NN}}(r)$ is given in Section 5. The implications of the present results for the description of non-resonant charge exchange and tunneling in dense plasmas are outlined in Section 6. The detailed treatment of the effect of the plasma environment on tunneling processes in plasmas, on effective statistical weights of bound states, and on the rates of kinetic processes will be presented in a separate paper [11]. In the present study, plasma is assumed to be classical, i.e., the temperature $T_{\mathrm{e}}$ of the free electrons is much higher than their Fermi energy.

\section{Nearest neighbor distribution}

We present here an analytical expression for $P_{\mathrm{NN}}(r)$ in nonideal classical plasmas. First, we derive the general, rigorous expressions for $P_{0}(r)$ and $P_{\mathrm{NN}}(r)$ as functionals of the potential $U_{\mathrm{NN}}(r)$ at the NN ion location, without making any assumptions on the form of the potential. Then, we find a simple semi-empirical analytical expression for $U_{\mathrm{NN}}(r)$ in a nonideal classical plasma. This allows us to obtain approximate expressions for $P_{0}(r)$ and $P_{\mathrm{NN}}(r)$ convenient for the actual calculations. Later in this work (in Sect. 4) we compare our theoretical predictions with the results of the MD simulations, in order to test the accuracy of the approximate expressions mentioned.

Let us denote by $Z_{\mathrm{t}}$ and $Z_{\mathrm{i}}$ the charges of the test ion and of the surrounding ions, respectively. We define the distance-to-the-NN-ion probability distribution $P_{\mathrm{NN}}(r) \mathrm{d} r$ as the probability to find the ion nearest to the test ion at the distance between $r$ and $r+\mathrm{d} r$ from the test ion. This is thus the probability for the absence of ions within the distance $r$ from the test ion and for the presence of an ion in the distance range $(r, r+\mathrm{d} r)$ from the test ion. The normalization condition is $\int_{0}^{\infty} P_{\mathrm{NN}}(r) \mathrm{d} r=1$. For brevity, we refer to $P_{\mathrm{NN}}(r)$ as the "NN distribution".

The common approach for obtaining $P_{\mathrm{NN}}(r)$ starts with the determination of the ion-ion pair correlation function $g(r)$, which gives the ratio of the (ensemble-averaged) density $n_{\mathrm{i}}(r)$ of the ions at the distance $r$ from the test ion to the density of the ions $\bar{n}_{\mathrm{i}}$ averaged over the macroscopic volume in consideration, i.e.,

$$
g(r)=n_{\mathrm{i}}(r) / \bar{n}_{\mathrm{i}}
$$

The pair correlation function in plasmas has been studied for several decades (see e.g. $[21,22]$ ). In reference [10] it was used for the theoretical determination of the NN distribution. The resulting expression for $P_{\mathrm{NN}}(r)$ was

$$
\begin{aligned}
P_{\mathrm{NN}}(r) & =4 \pi n_{\mathrm{i}}(r) r^{2} \exp \left\{-4 \pi \int_{0}^{r} n_{\mathrm{i}}\left(r^{\prime}\right) r^{\prime 2} \mathrm{~d} r^{\prime}\right\} \\
& =4 \pi \bar{n}_{\mathrm{i}} r^{2} g(r) \exp \left\{-4 \pi \bar{n}_{\mathrm{i}} \int_{0}^{r} r^{\prime 2} \mathrm{~d} r^{\prime} g\left(r^{\prime}\right)\right\} .
\end{aligned}
$$

However, even for accurate $g(r)$, this approach produces only approximate predictions for $P_{\mathrm{NN}}(r)$, the reason for the inaccuracy being the neglect of the effect on $n_{\mathrm{i}}(r)$ of the absence of ions inside $r$ (since the probability for the nearest ion to be found at $r$ is considered). The density of ions at $r$ under the constraint of absence of ions inside $r$ differs from $\bar{n}_{\mathrm{i}} g(r)$, and consequently the pair correlation function $g(r)$ cannot be used in the expression (1b) for a precise $P_{\mathrm{NN}}$ determination. A rigorous derivation, given in the Appendix, shows that the form (1a) is still accurate, provided $n_{\mathrm{i}}(r)$ is evaluated under the constraint of absence of ions inside $r$. For the proper choice of $n_{\mathrm{i}}(r)$ the equality between (1a) and (1b) is therefore only approximate.

The density $n_{\mathrm{i}}(r)$ of ions at the distance $r$ from the test ion, in the absence of other ions inside the sphere of radius $r$, can be evaluated using the Boltzmann formula for gases in potential fields [23]. Assuming that the ion distribution on coordinates and velocities outside $r$ corresponds to an equilibrium described by the ion temperature $T_{\mathrm{i}}$, the ion 
density $n_{\mathrm{i}}(r)$ at $r$ (i.e. between $r$ and $r+\mathrm{d} r$ ) is given by

$$
n_{\mathrm{i}}(r)=\bar{n}_{\mathrm{i}} \exp \left\{-\beta Z_{\mathrm{i}} e U_{\mathrm{NN}}(r)\right\},
$$

where $\beta=1 / T_{\mathrm{i}}$, and $U_{\mathrm{NN}}(r)$ is the total potential at the distance $r$ from the test ion. The subscript NN indicates that $U_{\mathrm{NN}}(r)$ is the potential experienced by the nearest neighbor ion at the distance $r$ from the test ion. This potential is produced primarily by the test ion (located at the origin) and by free electrons found in the sphere of radius $r$. Substituting the expression (2) for $n_{\mathrm{i}}(r)$ into expression (1a), we obtain

$$
\begin{aligned}
P_{\mathrm{NN}}(r)=4 \pi \bar{n}_{\mathrm{i}} r^{2} \exp \left\{-\beta Z_{\mathrm{i}} e U_{\mathrm{NN}}(r)\right. \\
\left.-4 \pi \bar{n}_{\mathrm{i}} \int_{0}^{r} r^{\prime 2} \mathrm{~d} r^{\prime} \exp \left(-\beta Z_{\mathrm{i}} e U_{\mathrm{NN}}\left(r^{\prime}\right)\right)\right\} .
\end{aligned}
$$

For the accurate potential $U_{\mathrm{NN}}(r)$ this expression is exact. The probability $P_{0}(r)$ to find no ions within $r$ from the test ion is given by the expression (A.1) derived in the Appendix. Expression (A.1) is also exact for the accurate $U_{\mathrm{NN}}(r)$.

Let us now find a reasonably accurate analytical approximation for $U_{\mathrm{NN}}(r)$. In this work we consider a nonideal classical plasma with the ion-ion coupling parameter

$$
\Gamma \equiv \Gamma_{\mathrm{ii}}=\beta Z_{\mathrm{i}}^{2} e^{2}\left(\frac{4}{3} \pi \bar{n}_{\mathrm{i}}\right)^{1 / 3}=\beta \frac{Z_{\mathrm{i}}^{2} e^{2}}{a}
$$

values in the range $0 \leq \Gamma<1$. Here

$$
a=\left(\frac{4}{3} \pi \bar{n}_{\mathrm{i}}\right)^{-1 / 3}
$$

is a characteristic interionic distance. We emphasize that the charges $Z_{\mathrm{i}}$ and $Z_{\mathrm{t}}$ are not the charges of the bare nuclei; rather, they include the charges of the bound electrons belonging to a plasma ion and to the test ion, respectively. This formulation of the problem allows the results to be readily applied to the problems of dense plasmas atomic physics, e.g., to the determination of the effective statistical weights of the bound states. Indeed, to determine the effective statistical weight of a certain quantum state of the test ion, one needs to know the distribution of the neighbor ions around the test ion in that state, i.e., in a specified ionization stage. The charge (the ionization stage) of both the test ion and the neighbor ions are therefore fixed as the external parameters of the problem. For now, we describe ions (including the test one) as pointlike particles. Thus, we neglect here the effects arising from the nonzero size of the ions (i.e., from the nonzero radius of the bound electron clouds). In Section 5 below, we discuss the accuracy of the pointlike ions assumption, and suggest correction terms for the NN ion potential. From the definitions of the distribution functions $P_{0}, P_{\mathrm{NN}}, P_{\mathrm{NNN}}$ it is clear that the quantities we are looking for are averaged over all the free electron coordinates. Therefore, we describe the free electrons as a continuous charge-density distribution rather than as individual particles.

To find the potential $U_{\mathrm{NN}}(r)$, it is convenient to start with the total ion-ion potential $U(r)$ in plasma. This is the total average potential produced by the test ion and by the surrounding ions and free electrons at a distance $r$ from the test ion. It is well known [22,24-27] that, as far as the total ion-ion potential is considered, the Debye-Hückel (DH) approximation is accurate for $\Gamma \lesssim 0.1$ and adequate for $0.1 \lesssim \Gamma<1$. Techniques that account for additional classes of correlation effects provide higher accuracy, but do not generally yield predictions for $U(r)$ in an analytic form [22] and are therefore unsuitable for our purpose. The total ion-ion potential $U(r)$ in the $\mathrm{DH}$ approximation is given by

$$
U(r)=\frac{Z_{\mathrm{t}} e}{r} \exp \left(-r / R_{\mathrm{S}}\right)
$$

where

$$
R_{\mathrm{S}}=\sqrt{\frac{T}{4 \pi e^{2}\left(Z_{\mathrm{i}}+Z_{\mathrm{i}}^{2}\right) \bar{n}_{\mathrm{i}}}}
$$

is the Debye screening radius. This expression for $R_{\mathrm{S}}$ assumes $T_{\mathrm{e}}=T_{\mathrm{i}}=T$ and macroscopic charge neutrality $\bar{n}_{\mathrm{e}}=Z_{\mathrm{i}} \bar{n}_{\mathrm{i}}$.

The potential $U_{\mathrm{NN}}(r)$ experienced by the NN ion contains, in comparison to $U(r)$, an additional term due to the uncompensated negative net charge of the free electrons within the sphere of radius $r$. We reiterate that, since all the test-ion bound electrons are already accounted for in the charge $Z_{\mathrm{t}}$ of the test ion, only the charge of the free electrons in the sphere $r$ must be accounted for explicitly in the derivation of $U_{\mathrm{NN}}(r)$. The free electrons do not concentrate within a small volume $V \ll \bar{n}_{\mathrm{e}}^{-1}$ around the test ion, but rather occupy the entire sphere $r$, and the free electron density $n_{\mathrm{e}}(r)$ does not diverge stronger than $r^{-2}$ near the origin. The nearest-neighbor potential $U_{\mathrm{NN}}(r)$ (as well as $U(r)$ ) at the small distances is therefore governed by the direct ion-ion repulsion, i.e.,

$$
U_{\mathrm{NN}}(r) \approx \frac{Z_{\mathrm{t}} e}{r} \text { for } r \ll \bar{n}_{\mathrm{i}}^{-1 / 3},
$$

and the exact asymptotic behavior of the free electron density at $r \rightarrow 0$ is thus unimportant. At the distances $r \gtrsim \bar{n}_{\mathrm{i}}^{-1 / 3}$, however, the effect of the free electrons charge inside $r$ is important. At these distances the two most important effects produced by the free electrons are (i) change in the net charge of the sphere $r$, which becomes in the first approximation $Z_{\mathrm{t}}-4 \pi r^{3} \bar{n}_{\mathrm{e}} / 3$, and (ii) screening of the net charge of the sphere $r$. Thus, we obtain

$$
U_{\mathrm{NN}}(r)=\frac{e}{r}\left(Z_{\mathrm{t}}-\frac{4}{3} \pi r^{3} \bar{n}_{\mathrm{e}}\right) \exp \left(-r / R_{\mathrm{S}}\right) .
$$

The semi-empirical expression for $U_{\mathrm{NN}}(r)$ above is, of course, approximate rather than exact. In particular, it is not a priori valid for $r \gg a$; however, as explained below, its validity is relevant only for $r$ as large as a few $a$. 
It is in order to test the validity of this expression (or, more exactly, of the expressions (3, A.1) evaluated using (5) for $U_{\mathrm{NN}}$ ) that we conduct the MD simulations the results of which are presented in Section 4.

Substituting the potential (5) into the general expression (3), one can see that, indeed, the characteristic spatial scale for $P_{\mathrm{NN}}(r)$ is given not by $R_{\mathrm{S}}$ but rather by a characteristic interionic distance $a$. The probability $P_{\mathrm{NN}}(r)$ is of practical interest only up to $r$ as large as a few $a$; for larger $r$ 's it is essentially zero. For $r>a$, even in ideal plasmas $P_{\mathrm{NN}}(r)$ converges with $r$ like $r^{2} \exp \left\{-(r / a)^{3}\right\}$. In nonideal plasmas the convergence at $r>a$ is faster yet, since at $r \approx a$ the total charge of the free electrons inside the sphere $r$ exceeds $-Z_{\mathrm{t}}$ and thus at larger $r$ the potential $U_{\mathrm{NN}}(r)$ is attractive for ions. The exception to this rule may be the case $Z_{\mathrm{t}} \gg Z_{\mathrm{i}}$, when $U_{\mathrm{NN}}(r)$ does not become attractive until roughly $r \approx a\left(Z_{\mathrm{t}} / Z_{\mathrm{i}}\right)^{1 / 3}$. The case $Z_{\mathrm{t}} \approx Z_{\mathrm{i}}$ is most common for plasmas. The case $Z_{\mathrm{t}} \gg Z_{\mathrm{i}}$, which may occur in plasmas of chemical mixtures, is of particular interest for stellar plasma physics. In the latter case, the test ion ("emitter ion") considered is often a multiple ion of a heavier element (say, Fe) immersed in a fully-ionized $\mathrm{H}$ plasma. In Section 4 , we compare $P_{\mathrm{NN}}$ given by expressions $(3,5)$ with the results of MD simulations. In our MD simulations electrons are presented as individual pointlike Coulomb particles, thus allowing the validity of our assumptions on $n_{\mathrm{e}}(r)$ to be tested.

\section{Distribution of the next-nearest neighbor ion}

The expression for the distribution $P_{\mathrm{NNN}}(\rho)$ of the distance $\rho$ from the test ion to the NNN ion is derived similarly to that for $P_{\mathrm{NN}}(r)$. Typically, the NNN ion has the same charge $Z_{\mathrm{i}}$ as the NN ion. The probability $P_{\mathrm{NNN}}(r, \rho) \mathrm{d} r \mathrm{~d} \rho$ for a NN ion to be found between $r$ and $r+\mathrm{d} r$ and for a NNN ion to be found between $\rho$ and $\rho+\mathrm{d} \rho$ is a product of three probabilities: (i) the probability $P_{\mathrm{NN}}(r) \mathrm{d} r$ for the $\mathrm{NN}$ ion to be found at the distance $r$, where $0 \leq r \leq \rho$, from the test ion; (ii) the probability $P_{0, \mathrm{NNN}}(r, \rho)$ to find no ions between $r$ and $\rho$ provided there are only two ions (test and NN) within the sphere of radius $r$; and (iii) the probability $P_{1, \mathrm{NNN}}(r, \rho) \mathrm{d} \rho$ to find an ion between $\rho$ and $\rho+\mathrm{d} \rho$ provided there are only two ions (test and NN) within $r$ and none between $r$ and $\rho$. Thus,

$$
P_{\mathrm{NNN}}(r, \rho)=P_{\mathrm{NN}}(r) P_{0, \mathrm{NNN}}(r, \rho) P_{1, \mathrm{NNN}}(r, \rho) .
$$

The probability for the NNN ion to be found between $\rho$ and $\rho+\mathrm{d} \rho$ (regardless of the $\mathrm{NN}$ ion position) is then given by

$$
\begin{aligned}
P_{\mathrm{NNN}}(\rho) & =\int_{0}^{\rho} P_{\mathrm{NNN}}(r, \rho) \mathrm{d} r \\
& =\int_{0}^{\rho} \mathrm{d} r P_{\mathrm{NN}}(r) P_{0, \mathrm{NNN}}(r, \rho) P_{1, \mathrm{NNN}}(r, \rho) .
\end{aligned}
$$

Here, $P_{\mathrm{NN}}(r)$ is given by expression (3). An expression for $P_{0, \mathrm{NNN}}(r, \rho)$, derived similarly to expression (A.1) of the Appendix, is

$$
\begin{aligned}
& P_{0, \mathrm{NNN}}(r, \rho)= \\
& \quad \exp \left\{-4 \pi \bar{n}_{\mathrm{i}} \int_{r}^{\rho} r^{\prime 2} \mathrm{~d} r^{\prime} \exp \left(-\beta Z_{\mathrm{i}} e U_{\mathrm{NNN}}\left(r, r^{\prime}\right)\right)\right\},
\end{aligned}
$$

where $U_{\mathrm{NNN}}\left(r, r^{\prime}\right)$ is the potential experienced by the NNN ion at $r^{\prime}$ (provided the $\mathrm{NN}$ ion is at $r$ ). The probability $P_{1, \mathrm{NNN}}(r, \rho)$ can be expressed via $U_{\mathrm{NNN}}(r, \rho)$ as

$$
P_{1, \mathrm{NNN}}(r, \rho)=4 \pi \rho^{2} \bar{n}_{\mathrm{i}} \exp \left(-\beta Z_{\mathrm{i}} e U_{\mathrm{NNN}}(r, \rho)\right) .
$$

To perform practical calculations, we suggest an expression for $U_{\mathrm{NNN}}(r, \rho)$ similar to that for $U_{\mathrm{NN}}(r)$ above (Eq. (5)), but with an account of two ions inside the sphere of radius $\rho$, i.e.,

$$
U_{\mathrm{NNN}}(r, \rho)=\frac{e}{\rho}\left(Z_{\mathrm{t}}+Z_{\mathrm{i}}-\frac{4}{3} \pi \rho^{3} \bar{n}_{\mathrm{e}}\right) \exp \left(-\rho / R_{\mathrm{S}}\right) .
$$

Note that this expression is independent of $r$. The accuracy of this expression and of the general form (7) will be discussed in Section 4. Using a procedure analogous to that described above, the distributions of the distances to the $3 \mathrm{rd}, 4 \mathrm{th}, \ldots, n$th nearest neighbor ions may be determined, too, if required.

Let us now derive the distribution $P_{\text {rat }}(\zeta)$ of the ratio $\zeta=\rho / r \geq 1$ of the distances. As a starting point we can take our expression for $P_{\mathrm{NNN}}(r, \rho) \mathrm{d} r \mathrm{~d} \rho$. For any given $r$, we are looking for the probability to find $\rho$ such that $\zeta r \leq$ $\rho<(\zeta+\mathrm{d} \zeta) r$. This probability is given by $P_{\mathrm{NNN}}(r, \rho) \mathrm{d} r \mathrm{~d} \rho$ for $\rho=\zeta r$ and $\mathrm{d} \rho=r \mathrm{~d} \zeta$, i.e. by

$$
P_{\mathrm{NNN}}(r, \zeta r) \mathrm{d} r \mathrm{~d} \rho=P_{\mathrm{NNN}}(r, \zeta r) r \mathrm{~d} r \mathrm{~d} \zeta .
$$

Since a given value of $\zeta$ can occur for any value of $r$,

$$
P_{\mathrm{rat}}(\zeta) \mathrm{d} \zeta=\int_{r=0}^{\infty} P_{\mathrm{NNN}}(r, \zeta r) r \mathrm{~d} r \mathrm{~d} \zeta
$$

This expression is valid for both ideal and nonideal plasmas. For an ideal plasma, $\beta \rightarrow 0$, the expression for $P_{\text {rat }}(\zeta) \mathrm{d} \zeta$ acquires a particularly simple form. In this limit,

$$
\begin{aligned}
P_{\mathrm{NN}}(r) & =4 \pi \bar{n}_{\mathrm{i}} r^{2} \exp \left(-\frac{4}{3} \pi \bar{n}_{\mathrm{i}} r^{3}\right) \\
& =3 \frac{r^{2}}{a^{3}} \exp \left(-\frac{r^{3}}{a^{3}}\right) \\
P_{0, \mathrm{NNN}}(r, \rho) & =\exp \left(-\frac{\rho^{3}-r^{3}}{a^{3}}\right) \\
P_{1, \mathrm{NNN}}(r, \rho) & =3 \frac{\rho^{2}}{a^{3}}
\end{aligned}
$$

and thus

$$
P_{\mathrm{NNN}}(r, \zeta r)=9 \frac{\zeta^{2} r^{4}}{a^{6}} \exp \left(-\frac{\zeta^{3} r^{3}}{a^{3}}\right) .
$$


For the $\zeta$ distribution in an ideal plasma, the integration (11) gives:

$$
P_{\text {rat }}^{\mathrm{id}}(\zeta) \mathrm{d} \zeta=\frac{3}{\zeta^{4}} \mathrm{~d} \zeta
$$

This is, however, the case only in the strictly ideal plasmas. Even in the weakly-coupled plasmas one finds $P_{\text {rat }}(\zeta)$ to decay exponentially as $\zeta \rightarrow \infty$. It is, indeed, easy to see that for large $\zeta$ the main contribution to the integral (11) comes from small $r$. The reason for the power-law decay of $P_{\mathrm{rat}}^{\mathrm{id}}(\zeta)$ at large $\zeta$ is therefore in the slow convergence of $P_{\mathrm{NN}}(r)$ as $r \rightarrow 0$ in the ideal plasmas. In other words, when $\rho / r$ is large it is with much higher probability due to $r \ll a$ than due to $\rho \gg a$. Now, we note that even in the weakly-coupled plasmas the decay of $P_{\mathrm{NN}}(r)$ at $r \rightarrow 0$ is actually exponential, due to the direct Coulomb repulsion between the test ion and the NN ion. The decay of $P_{\mathrm{NN}}(r)$ at $r \rightarrow 0$ is exponential even when the test ion is neutral, as at the sufficiently small $r$ the NN ion penetrates the bound electrons cloud of the test ion (see Sect. 5). Therefore, it is only in the formal limit of the strictly ideal plasma that $P_{\text {rat }}(\zeta)$ decays at $\zeta \rightarrow \infty$ according to the power law. In real plasmas, at sufficiently large values of $\zeta$ the decay always becomes exponential. The smaller is $\Gamma_{\mathrm{ii}}$, the larger are the values of $\zeta$ at which this occurs. In the opposite limit of a very strong coupling $\left(\Gamma_{\mathrm{ii}}>150[10,22]\right)$ one should expect the ions to settle into a crystal lattice, and $P_{\text {rat }}(\zeta)$ to tend to a delta-function at $\zeta=1$.

The distribution function $P(\theta)$ of the angle $\theta$ between the directions to the $\mathrm{NN}$ ion and to the $\mathrm{NNN}$ ion in an ideal plasma is readily obtained. We choose the direction from the test ion to the $\mathrm{NN}$ ion as the $z$-direction, relative to which the angle $\theta$ is defined, $0 \leq \theta \leq \pi$. We define $P(\theta, \varphi) \mathrm{d} \Omega$ as the probability to find the direction to the NNN ion in the element of the solid angle $\mathrm{d} \Omega=$ $\sin \theta \mathrm{d} \theta \mathrm{d} \varphi$. The symmetry on $\varphi$ implies $P(\theta, \varphi) \mathrm{d} \Omega \equiv$ $(1 / 2 \pi) P(\theta) \sin \theta \mathrm{d} \theta \mathrm{d} \varphi$. In ideal plasmas the distribution is uniform over $\Omega$, therefore $P^{\mathrm{id}}(\theta)=1 / 2$ and thus the probability to find the NNN ion between $\theta$ and $\theta+\mathrm{d} \theta$ in ideal plasmas is given by $\sin \theta \mathrm{d} \theta / 2$. In non-ideal plasmas the repulsion between the NN and NNN ions becomes significant, and $P(\theta)$ decreases as $\theta$ approaches 0 . This deviation from the distribution uniformity over $\Omega$ is examined by MD simulations, and the results are presented in the next section.

\section{MD simulations}

We have performed classical-trajectory molecular-dynamics simulations for two-component electron-ion plasma. Both electrons and ions were treated as classical nonrelativistic pointlike particles with masses $m_{\mathrm{e}}$ and $M_{\mathrm{i}}$ and charges $-e$ and $e$, respectively. The charge neutrality condition was given by the equation $N_{\mathrm{e}}=N_{\mathrm{i}}$, where $N_{\mathrm{e}}$ and $N_{\mathrm{i}}$ are the total numbers of electrons and ions considered. The initial distribution of the particles inside the modeling volume $V$ was uniform (random for each particle, and uncorrelated). The initial velocity distribution of the particles was Maxwellian, with the electron and ion temperatures taken to be equal, i.e., $T_{\mathrm{e}}=T_{\mathrm{i}}=T$.

To speed up the simulations, the ion mass was reduced to $M_{\mathrm{i}}=10 \mathrm{~m}_{\mathrm{e}}$. It is easy to see that in equilibrium the near-neighbor distributions do not depend on the ratio $M_{\mathrm{i}} / m_{\mathrm{e}}$. The simulated near-neighbor distributions, in comparison to the simulated local microfield distributions, are significantly less sensitive to the total number of particles used in the MD simulations. Still, we ascertained that for every set of parameters the linear size of the simulated plasma volume was much larger than the Debye radius, in order to avoid any ambiguity in the interpretation of the results. In a typical run, 1260 particles were simulated. Larger particle numbers were used in the simulations at lower $\Gamma$ values. In several cases we have repeated the tests with a twice the number of particles simulated. The results for single and double particle numbers were, within the noise range, indistinguishable. Lack of need to simulate large numbers of particles allowed us to use a mirror walls approximation for the boundaries of the simulated volume (instead of the periodic boundaries commonly used). The finite-differences algorithm used to solve the equations of motion was based on a second-order scheme with adaptive time-step. This approach proved reliable enough to keep the plasma temperature nearly constant throughout the run, i.e., for tens of ion plasma periods $\tau_{\mathrm{pi}}=2 \pi \sqrt{M_{\mathrm{i}} /\left(4 \pi \bar{n}_{\mathrm{i}} Z_{\mathrm{i}}^{2} e^{2}\right)}$. Since the classical description was used for electron-ion interactions, the capture of electrons into discrete atomic quantum states was not possible. To avoid the classical "Coulomb collapse", the electron-ion Coulomb interaction was cut off exponentially at small distances. At the end of the present section we examine the effect of this cutoff in detail.

We have conducted MD simulations for a series of $\Gamma$ values between 0.015 and 0.79 . At the ion density $\bar{n}_{\mathrm{i}}=10^{18} \mathrm{~cm}^{-3}$ and $Z_{\mathrm{i}}=Z_{\mathrm{t}}=1$ used, these values of $\Gamma$ correspond to ion temperatures $T_{\mathrm{i}}$ between 15.2 and $0.294 \mathrm{eV}$. As we said above, our analytical expressions for the spatial distribution functions, which are using (5) for $U_{\mathrm{NN}}(r)$, become exact in the limit of small $\Gamma$. At $\Gamma=0.015$, the results of the theory and of the MD simulations are practically indistinguishable. Figure 1 shows our theoretical predictions for $P_{\mathrm{NN}}(r)$ and $P_{\mathrm{NNN}}(\rho)$ compared to the results of MD simulations, for several $\Gamma$ values. The distances $r$ and $\rho$ are given in units of $a$, and the distribution functions $P_{\mathrm{NN}}(r)$ and $P_{\mathrm{NNN}}(\rho)$ are given in units of $a^{-1}$. Let us first consider the comparison results for $P_{\mathrm{NN}}$. One can see that the agreement is excellent, to within $1 \%$ or better near the peak. The predicted relative level of statistical noise for the MD results is slightly less than $1 \%$ in the vicinity of the peak, growing to few per cent on the wings. For $\Gamma=0.015$, the theoretical and MD results coincide within the noise levels. For $\Gamma=0.10$, the theoretical and MD results coincide within the noise levels except in the region $0.2 a<r<0.6 a$, where the MD results are $1-3 \%$ higher than the theory predicts, which is slightly outside the noise range. For $\Gamma=0.35$ the MD results coincide with the theory predictions in the peak region $0.6 a<r<1.3 a$, exceed somewhat the theory 


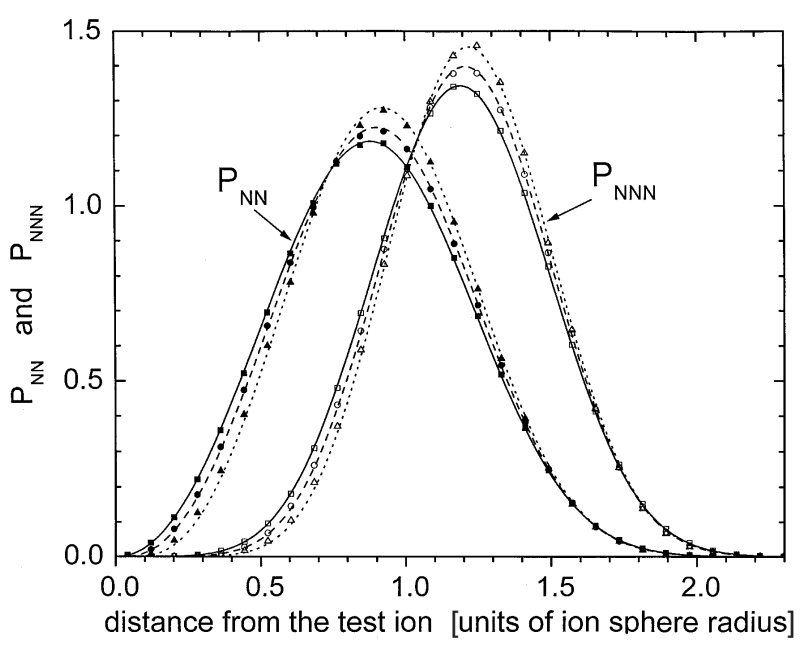

Fig. 1. Comparison of the MD results for $P_{\mathrm{NN}}(r)$ and $P_{\mathrm{NNN}}(\rho)$ with the analytical predictions. MD results are shown for $\Gamma=$ 0.015 by squares, for $\Gamma=0.10$ by circles, and for $\Gamma=0.35$ by triangles. Solid and hollow symbols show results for $P_{\mathrm{NN}}$ and $P_{\mathrm{NNN}}$, respectively. Theoretical predictions are shown by the solid lines for $\Gamma=0.015$, by the dashed lines for $\Gamma=0.10$, and by the dotted lines for $\Gamma=0.35$.

predictions in the region $0.2 a<r<0.6 a$, and are slightly below the theory predictions in the region $r>1.3 a$ (roughly 2-3 times above the noise level). We have found that the discrepancy in the $0.2 a<r<0.6 a$ region at larger $\Gamma$ 's is due to the effect of the classical quasiatoms in the MD simulations, as it will be explained at the end of the present section. The discrepancy in this case is caused by the assumptions made in the MD model rather than by the inaccuracy in the theoretical treatment. The discrepancy for $\Gamma=0.35$ and $r>1.3 a$, on the other hand, is due to both the local density variations in the MD model and the limited accuracy of our approximation for $U_{\mathrm{NN}}(r)$ at these $\Gamma$. For the distribution $P_{0}(r)$ the agreement between the theory and the MD simulation results is better yet. Indeed, $P_{0}(r)$ is mostly influenced by the near-peak values of $P_{\mathrm{NN}}(r)$, for which the agreement is excellent.

The agreement of the theoretical predictions for $P_{\text {NNN }}(\rho)$ with the MD simulation results is also very good, even surprising, considering the simplicity of the assumptions we made for $U_{\mathrm{NNN}}$. Indeed, in general, the probability to find the NN ion at $\mathbf{r}$ and the NNN ion at $\boldsymbol{\rho}$ not only depends on the absolute values of $\mathbf{r}$ and $\boldsymbol{\rho}$ but also on the angle $\theta$ between their directions. The probabilities $P_{0, \mathrm{NNN}}(r, \rho)$ and $P_{1, \mathrm{NNN}}(r, \rho)$ in expression $(7)$ are implicitly integrated over $\theta$. When the integration over $\theta$ is carried out, the effect of the higher-than-monopole terms in the expansion of the total potential around the origin on $P_{0, \mathrm{NNN}}(r, \rho)$ and $P_{1, \mathrm{NNN}}(r, \rho)$ does not vanish completely. Thus, in contrast to expressions $(3,6,7,11)$ for $P_{\mathrm{NN}}(r), P_{\mathrm{NNN}}(r, \rho), P_{\mathrm{NNN}}(\rho)$, and $P_{\text {rat }}(\zeta)$, respectively, which are all exact, expressions $(8,9)$ for $P_{0, \mathrm{NNN}}(r, \rho)$ and $P_{1, \mathrm{NNN}}(r, \rho)$ are approximate. Indeed, it is easier for the NNN ion to approach the test ion from the side opposite to $\mathrm{NN}$ ion, and harder to approach from the same side; these two effects do not a priori cancel each-other out as a re- sult of the integration over $\theta$, and must be accounted for if an exact expression for the product $P_{0, \mathrm{NNN}} P_{1, \mathrm{NNN}}(r, \rho)$ for nonideal plasmas is to be written. Still, for $\Gamma<1$ the accuracy of the prediction $(7-10)$ for $P_{\mathrm{NNN}}(\rho)$ is very good.

In order to test the model validity for larger ion charges, we have also performed simulations for $Z_{\mathrm{i}}=Z_{\mathrm{t}}=$ 3 . In this case, the electroneutrality dictated $N_{\mathrm{e}}=3 N_{\mathrm{i}}$; specifically, 500 ions and 1500 electrons were simulated, for $\Gamma_{\mathrm{ii}}$ values of 0.16 and 0.33 . Note that for $Z_{\mathrm{i}} \neq 1$ the coupling constants depend on the particle types, namely, $\Gamma \equiv \Gamma_{\mathrm{ii}}=Z_{\mathrm{i}} \Gamma_{\mathrm{ie}}=Z_{\mathrm{i}}^{2} \Gamma_{\mathrm{ee}}=\beta Z_{\mathrm{i}}^{2} e^{2} / a$. Our theoretical calculations agree very well with the MD simulation results in this case, too.

The MD simulations described here also produce the distribution of the ratio $\zeta=\rho / r$ of the distances to the NNN and NN ions. Figure 2a presents the comparison of the MD results for $P_{\text {rat }}(\zeta)$ with the ideal-plasma prediction (12) shown by the solid line. For clarity, statistical error bars are only shown for MD data at $\Gamma=0.35$. One can see that the tail of the $P_{\text {rat }}(\zeta)$ distribution diminishes with the increase of $\Gamma$, as we predicted. Still, for $\Gamma<1$ the deviations of $P_{\text {rat }}(\zeta)$ from $P_{\text {rat }}^{\text {id }}(\zeta)$ are small, especially in the $\zeta \sim 1$ region. For example, as $\Gamma$ increases from 0 to 0.35 , the average $\langle\zeta\rangle$ decreases by about $6 \%$ only. Figure $2 \mathrm{~b}$ shows the same $\Gamma=0.35$ data set as Figure 2a, but in the range $1<\zeta \leq 2$ and in a linear scale for $P_{\text {rat }}(\zeta)$. The ideal plasma limit is also shown. The two data sets for smaller $\Gamma$ 's shown in Figure 2a are omitted in Figure $2 \mathrm{~b}$ as they lie too close to $P_{\text {rat }}^{\text {id }}(\zeta)$. One can see that even for $\Gamma=0.35$ the difference between the MD results and the ideal-plasma prediction for $P_{\text {rat }}(\zeta)$ in the region $1<\zeta<1.7$ is less than $5 \%$.

The probability $P(\theta)$ to find a certain angle $\theta$ between the directions to the NN and to the NNN ions is shown in Figure 3. The MD results for $\Gamma=0.35,0.075$, and 0.015 are presented, as well as the ideal plasma limit $P^{\text {id }}(\theta)=1 / 2$. As noted above, the probability to find a certain value of $\theta$ in the small interval $\mathrm{d} \theta$ is given by $P(\theta) \sin \theta \mathrm{d} \theta$. For all three sets of MD data shown, the relative statistical error was approximately $2 \%$. The error bars are only shown for $\Gamma=0.35$ data; error bars for other $\Gamma$ values are similar. Figure 3 demonstrates that in nonideal plasmas with $\Gamma<1$, the probability to find small values of the angle $\theta$ is reduced due to the repulsion between the NN and NNN ions, whereas for $\theta \gtrsim \pi / 3$ the distribution $P(\theta)$ remains practically uniform, confirming the intuitive expectation. As $\Gamma$ increases from 0 to 0.35 , it is seen that, first, the probability to find small values of $\theta$ decreases, and, second, the cone around the direction towards the NN ion, in which $P(\theta)$ is reduced, becomes wider. However, note that due to the $\sin \theta$ factor in $\mathrm{d} \Omega$, the most probable values of $\theta$ are around $\pi / 2$. This remains the case throughout our $\Gamma$ range, despite the repulsive force exerted by the $\mathrm{NN}$ ion.

Let us now discuss the limitations of the MD model arising from the classical description of the electrons, i.e., from the neglect of the quantum effects in the short-range electron-ion interaction. It is well known (see, e.g., [28]) 


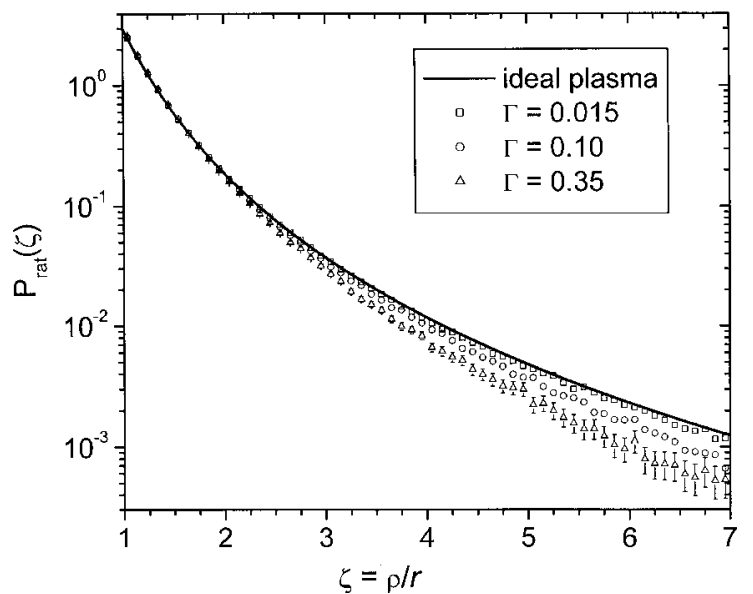

(a)

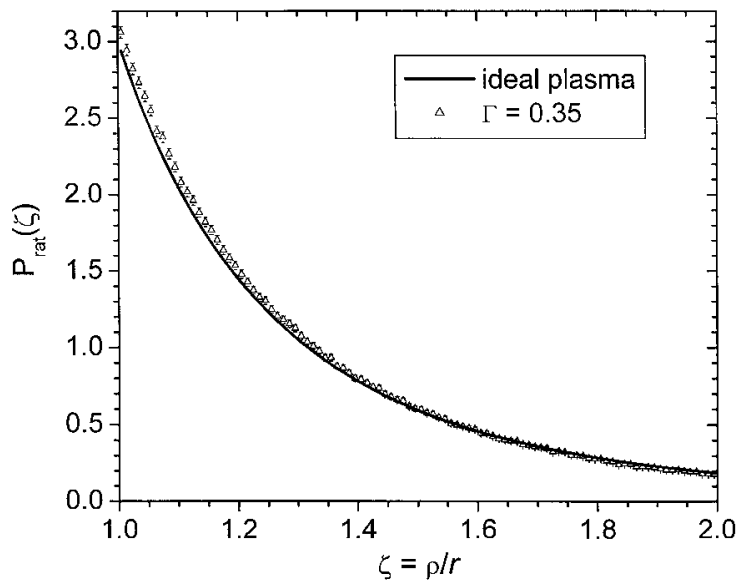

(b)

Fig. 2. (a) Comparison of the MD results for $P_{\text {rat }}(\zeta)$ with the ideal-plasma predictions. (b) The same data as in (a), in the $1<\zeta \leq 2$ region, shown on linear scale.

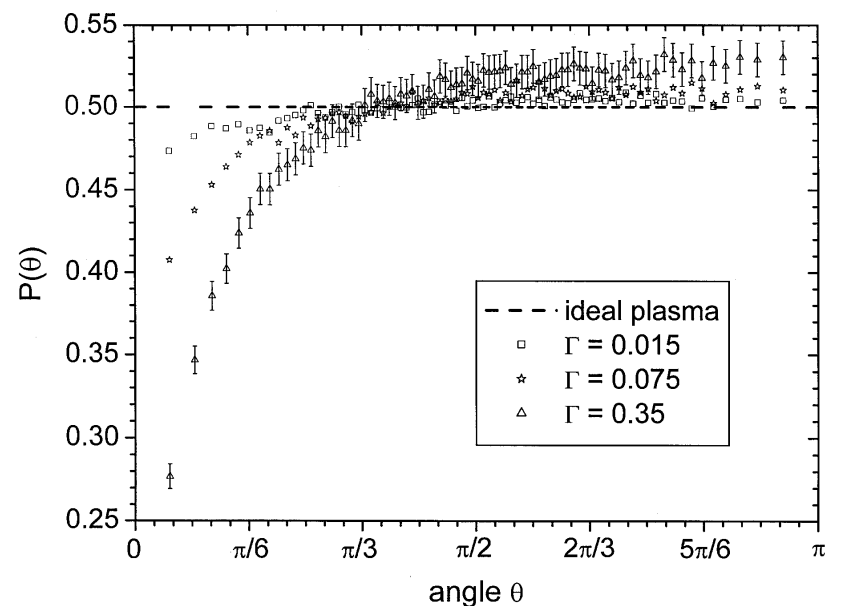

Fig. 3. Comparison of the MD results for $P(\theta)$ with the idealplasma predictions. that, to avoid the collapse of the classical system of electrons and ions, the direct electron-ion interaction potential

$$
U_{\mathrm{ei}}(r)=\frac{Z_{\mathrm{i}} e}{r}
$$

must be replaced by the pseudopotential with a "regularized" short-range behavior. We have chosen

$$
\widetilde{U}_{\mathrm{ei}}(r)=\frac{Z_{\mathrm{i}} e}{r}\left(1-\exp \left\{-r / \lambda_{\mathrm{c}}\right\}\right)
$$

where the cutoff radius $\lambda_{\mathrm{c}}$ may be qualitatively thought of as a classical emulation of the electron de Broglie length. The regularized potential $\widetilde{U}_{\text {ei }}(r)$ converges to $U_{\text {ei }}(r)$ exponentially at $r \gg \lambda_{c}$. In the opposite limit, $r \rightarrow 0$ (i.e., $\left.r \ll \lambda_{\mathrm{c}}\right), \widetilde{U}_{\mathrm{ei}}(r)$ approaches the constant value $\widetilde{U}_{\mathrm{ei}}^{\mathrm{min}}=$ $Z_{\mathrm{i}} e / \lambda_{\mathrm{c}}$. We note that, according to the formulation of the problem we have given in the Section 2, the numbers of bound electrons in the test ion and in the $\mathrm{NN}$ ion are the external parameters of the model. We do not consider the spatial distribution of the bound electrons; rather, their charges are included in $Z_{\mathrm{i}}$ and $Z_{\mathrm{t}}$. Only the free electrons spatial distribution should be accounted for explicitly in the potential $U_{\mathrm{NN}}(r)$. Therefore, it is important to make sure that in the MD simulations all of the electrons (or at least most of them) are indeed free rather than found in the classical bound states. The MD-simulated electrons occupying the quasi-periodical orbits with $r \ll a$ around individual ions can be for all practical purposes considered bound. In the case when a significant fraction of all simulated electrons (in the ensemble-average sense) is found in the bound quasi-periodical orbits, the MD simulations obviously do not correspond to the formulation of the problem quoted above, since the simulated $Z_{\mathrm{i}}$ and $Z_{\mathrm{t}}$ are effectively reduced. Thus, the parameter $\lambda_{c}$ must be chosen large enough for the relative abundance of the "classical bound electrons" to be much smaller than unity. On the other hand, the parameter $\lambda_{\mathrm{c}}$ must be chosen small enough so not to affect the free electron density at $r \sim a$, thus, for the simulations to be accurate, the condition $\lambda_{\mathrm{c}} \ll a$ must be obeyed. Let us now consider the choice of $\lambda_{\mathrm{c}}$ in greater detail.

We are looking for a constraint on $\lambda_{\mathrm{c}}$ imposed by the requirement of a low relative abundance of the classical bound electrons in a simulated plasma of ions of a charge $Z_{\mathrm{i}}$. For the classical electron found over the lowest part of the $\widetilde{U}_{\text {ei }}(r)$ well (i.e., within $r \lesssim \lambda_{\text {c }}$ from an ion) the available volume is $4 \pi \lambda_{\mathrm{c}}^{3} / 3$, while for a free electron it is $\bar{n}_{\mathrm{e}}^{-1}=4 \pi a^{3} Z_{\mathrm{i}} / 3$. The loss of the available volume is compensated, for a bound electron, by the increased probability to be found in that volume, i.e., by the Boltzmann factor $\exp \left\{\beta_{\mathrm{e}} e \widetilde{U}_{\mathrm{ei}}^{\mathrm{min}}\right\} \equiv \exp \left\{\Gamma_{\mathrm{ei}} a / \lambda_{\mathrm{c}}\right\}$, where $\beta_{\mathrm{e}}$ is the inverse electron temperature. Thus, the relative "statistical weight" of the electron states within $r \lesssim \lambda_{\mathrm{c}}$ from ions is estimated by

$$
W \approx Z_{\mathrm{i}}\left(\frac{\lambda_{\mathrm{c}}}{a}\right)^{3} \exp \left\{\Gamma_{\mathrm{ei}} \frac{a}{\lambda_{\mathrm{c}}}\right\},
$$


and the fraction of the electrons found on average within $r \lesssim \lambda_{\mathrm{c}}$ from the ions is then given by

$$
Q=\frac{W}{W+1}
$$

Note that $N_{\mathrm{e}} Q$ is not strictly the number of classicallybound electrons, but rather a total number of electrons found within $r \lesssim \lambda_{\mathrm{c}}$. However, since $\lambda_{\mathrm{c}} \ll a$, it is certain that the fraction of the free electrons within $r \lesssim \lambda_{\mathrm{c}}$ is small, and thus whenever $Q$ becomes comparable to 1 it is mostly due to the bound electrons. In other words, the MD simulations are valid as long as $Q \ll Z_{\mathrm{i}}^{-1}$, i.e., as long as the effective reduction in $Z_{\mathrm{i}}$ is much smaller than unity. From equations $(14,15)$ it is clear that for $\Gamma_{\text {ei }} a / \lambda_{\mathrm{c}} \ll 1$ the ion potential wells (13) are too shallow to keep bound electrons inside at the temperatures considered, and $Q$ reduces indeed to a trivial geometrical factor $Z_{\mathrm{i}}\left(\lambda_{\mathrm{c}} / a\right)^{3} \ll 1$. In the opposite case of a deep well, $\Gamma_{\mathrm{ei}} a / \lambda_{\mathrm{c}} \gg 1$, which can be due to a small $\lambda_{\mathrm{c}}$ (Coulomb collapse) and/or to a large $\Gamma_{\text {ei }}$ (strong coupling), most of the electrons are "classically bound", $Q \approx 1$. Note that in the case when $Z_{\mathrm{i}} \gg 1$, the first electrons to collapse onto Kepler orbits reduce the effective $Z_{\mathrm{i}}$ and $\Gamma_{\mathrm{ei}}$ for the next ones, so a significant fraction of electrons can stay free then even for $\Gamma_{\mathrm{ei}} a / \lambda_{\mathrm{c}} \gg 1$; but since $Z_{\mathrm{i}}$ is effectively reduced, the MD results in that case are still inapplicable for our purpose. The minimal value of $W$,

$$
W^{\min }=Z_{\mathrm{i}}\left(\frac{\Gamma_{\mathrm{ei}}}{3}\right)^{3} \exp (3) \approx 0.744 Z_{\mathrm{i}} \Gamma_{\mathrm{ei}}^{3},
$$

occurs at $\lambda_{\mathrm{c}}=\Gamma_{\mathrm{ei}} a / 3$. It is thus clear that for $\Gamma_{\mathrm{ei}} \gtrsim 0.3$ the capture of the electrons onto classical orbits becomes an important issue, and for $\Gamma_{\text {ei }} \gtrsim 0.5$ the significant contribution from the classically bound electrons cannot be avoided in the MD models with pointlike classical electrons; while it is only such models that can be utilized to test consistently the free electrons effect on the ion-ion spatial correlations. For that reason, we do not consider it amenable to present here the results of the MD simulations for $\Gamma_{\mathrm{ei}} \gtrsim 0.5$. Still, we note that the simulation results for $Z_{\mathrm{i}}=1, \Gamma \approx 0.8$ agree well with the theoretical predictions.

\section{Discussion}

In the present work, (i) we have derived the exact expressions for the functions $P_{\mathrm{NN}}(r)$ and $P_{0}(r)$ in terms of the average potential $U_{\mathrm{NN}}(r)$ at the $\mathrm{NN}$ ion location, and for the functions $P_{\mathrm{NNN}}(r, \rho), P_{\mathrm{NNN}}(\rho)$, and $P_{\text {rat }}(\zeta)$ in terms of the function $P_{\mathrm{NN}}(r)$ and of the product $P_{0, \mathrm{NNN}} P_{1, \mathrm{NNN}}(r, \rho)$; (ii) we have found the semi-empirical expressions for the potentials $U_{\mathrm{NN}}(r)$ and $U_{\mathrm{NNN}}(r, \rho)$ valid for ion-ion coupling parameter values $0 \leq \Gamma<1$, and used those expressions to find closed analytical expressions for the distribution functions above; and, finally, (iii) we have conducted the MD simulations, the results of which are found in excellent agreement with the approximate analytical expressions derived above. The simulations utilize the pure Coulomb particle-particle interaction potentials regularized at close range, and are free from the assumptions made to find $U_{\mathrm{NN}}$ and $U_{\mathrm{NNN}}$. This supports our arguments behind the semi-empirical expressions $(5,10)$ for $U_{\mathrm{NN}}(r)$ and $U_{\mathrm{NNN}}(r, \rho)$, respectively, and suggests that equations $(5,10)$ indeed approximate well the true functions $U_{\mathrm{NN}}(r)$ and $U_{\mathrm{NNN}}(r, \rho)$ in the plasmas with $0 \leq \Gamma<1$, within the framework of the pointlike ions model. More specifically, obtaining the expressions for $U_{\mathrm{NN}}(r)$ and $U_{\mathrm{NNN}}(r, \rho)$, we have (a) assumed that both the test ion and the perturber ions are pointlike, and (b) assumed a particular form of the screening contribution to the NN and NNN potentials $(5,10)$. The direct comparisons to the MD simulations data suggest that the second assumption is quite accurate at the $\Gamma$ values considered. We emphasize again that in the MD simulations both electrons and ions were represented as pointlike Coulomb charges, thus all the screening effects were produced in the MD simulations by the self-consistent particle motion, and not introduced artificially.

Thus, to complete the rigorous analysis of the neighbor ion distribution functions in the plasmas considered, we would like now to consider in detail the precision of the pointlike ions assumption lying at the base of the present model for $U_{\mathrm{NN}}(r)$ and $U_{\mathrm{NNN}}(r, \rho)$. To estimate quantitatively the error arising from the use in expressions $(5,10)$ of the pointlike ions approximation, we must forgo the generality of the present formulation of the problem, and turn to a detailed study of the individual atomic states at the plasma parameter values that are relevant for the particular applications of the results above. The problems to the treatment of which the present results can be applied directly are, for example, the collectivization of the bound states in plasmas, determination of the occupation factors and of the corresponding effective statistical weights of the atomic and ionic states, spectroscopy and statistics of the transient quasimolecular states and of bound states in dense plasmas in general. Thus, we consider the test atom or ion in an excited state (ground states are rather weakly affected by the density effects even at $\Gamma=1$ ). The perturber ions, on the other hand, are usually found to be in the ground state. As we said in Section 2, perturber ions typically have the charge similar or smaller than that of the test ion; the latter case is realized when the dominant chemical element in plasma is fully ionized. The characteristic linear size of an ion in an excited state exceeds significantly the size of a similar-charge ion in the ground state. Thus, perturber ions may well be considered pointlike in comparison to the test ion, even when they are not fully ionized. Consequently, below we will be concerned primarily with the effects of the nonzero size of the emitter ion, or, more specifically, with the effects of the nonzero characteristic radius of the emitter ion optical electron wavefunction.

It is well known (see, e.g., [20]) that the certain bound state $q$ of the emitter ion undergoes an overbarrier ionization when the distance between the emitter ion and its 
$\mathrm{NN}$ ion is smaller than

$$
r_{q}=\frac{Z_{0} e^{2}}{E_{q}}\left\{1+2\left(\frac{Z_{\mathrm{i}}}{Z_{0}}\right)^{1 / 2}\right\},
$$

where $Z_{0} \equiv Z_{\mathrm{t}}+1$ is the charge of the emitter parent ion, and $E_{q}$ is the binding energy of the emitter ion optical electron in the bound state $q$. The characteristic linear size of the emitter ion, defined by the radius of the classicallyallowed region for the optical electron in the potential well of the emitter parent ion, is given by

$$
R_{q}=\frac{Z_{0} e^{2}}{E_{q}}
$$

Note that $R_{q}$ is slightly larger than the state $q$ average wavefunction radius, which could be utilized alternatively as the atomic linear size scale. The meaning of the scales $r_{q}$ and $R_{q}$ is as follows. The ion densities of interest are those producing $a \gtrsim r_{q}$, otherwise the occupation factor (i.e., the surviving bound fraction) for the state $q$ is much less than unity, and the state $q$ is virtually "extinct". The charge of the test ion, on the other hand, may be considered equal to $Z_{\mathrm{t}}$ as long as $r \gg R_{q}$. As $r$ approaches $R_{q}$, the test ion charge starts to grow, and approaches $Z_{0}=Z_{\mathrm{t}}+1$ for $r \ll R_{q}$ (as the $\mathrm{NN}$ ion penetrates the "orbit" of the test ion optical electron). It is thus clear that the principal amendment for $U_{\mathrm{NN}}(r)$ in this situation is to replace $Z_{\mathrm{t}}$ in (5) by a smooth function $\widetilde{Z}_{\mathrm{t}}(r)$, changing from $Z_{\mathrm{t}}$ to $Z_{\mathrm{t}}+1$ as $r$ becomes smaller than $R_{q}$. Note that this implies that $P_{\mathrm{NN}}(r)$ always converges to zero exponentially at small $r$, even in the case when $Z_{\mathrm{t}}=0$ (neutral emitter). As $r$ decreases further, it is possible that the NN ion would also penetrate the orbits of the emitter ion core electrons, corresponding to a further increase in $\widetilde{Z}_{\mathrm{t}}(r)$; but this occurs at $r$ 's too small to be important for practical purposes. It is clear from the comparison of $r_{q}$ and $R_{q}$ that the plasmas with $Z_{\mathrm{t}} \gg Z_{\mathrm{i}}$ and the plasmas with $Z_{\mathrm{t}} \approx Z_{\mathrm{i}}$ differ qualitatively with the respect to the spatial scales relation involved. Indeed, in the plasmas with $Z_{\mathrm{t}} \approx Z_{\mathrm{i}}$, the bound state in question becomes free (moves above the barrier peak) when the distance to the NN ion, $r=r_{q}$, is significantly larger than the emitter ion size $R_{q}$ (typically, by a factor of 3 or so). Then, for the situation of interest, $r_{q} \lesssim a$, only the low- $r$ wing of the distributions $P_{0}(r), P_{\mathrm{NN}}(r)$ and $P_{\mathrm{NNN}}(r)$ may be affected by the increase in the charge of the emitter ion as seen by its near neighbors. $P_{0}(r)$ is particularly insensitive to this effect, since in the region $r \lesssim a / 3$ one finds $P_{0}(r) \approx 1$ anyway. The situation in the plasmas where $Z_{\mathrm{t}} \gg Z_{\mathrm{i}}$ is quite different, since in those plasmas the ratio $r_{q} / R_{q}$ is notably smaller (often smaller than 2), and an overbarrier escape of the optical electron does not occur unless the nearest perturber ion gets almost as close to the emitter ion as $r=R_{q}$. On the other hand, for $Z_{\mathrm{t}} \gg 1$ the change of $Z_{\mathrm{t}}$ by 1 does not produce a significant effect on the neighbor ions spatial distribution.

The maximal value of the ion density $\bar{n}_{\mathrm{i}}$ for which our model for $U_{\mathrm{NN}}(r)$ and $U_{\mathrm{NNN}}(r, \rho)$ is still valid is given by the condition $\Gamma_{\mathrm{ii}}<1$, i.e.,

$$
\bar{n}_{\mathrm{i}}<\frac{3 T^{3}}{4 \pi Z_{\mathrm{i}}^{6} e^{6}} .
$$

The account of the nonzero size of the perturber ions does not impose an additional limitation on $\bar{n}_{\mathrm{i}}$, as for $\Gamma_{\mathrm{ii}}<1$ the interionic distance $a$ exceeds the average radius of a perturber ion $\left\langle r_{\mathrm{gs}}\right\rangle$ at least by a factor of 3 . Indeed, the ionization composition of dense plasmas (except the extremely transient ones) is close to that in the local thermodynamic equilibrium. Then, ions are found predominantly in the ionization stage with the ground state binding energy $E_{\mathrm{gs}} \approx 6 T_{\mathrm{e}}$, as the system of Saha equations readily shows (see e.g. [29]). Condition $\Gamma_{\mathrm{ii}}<1$ rewrites then as

$$
a>\frac{6 Z_{\mathrm{i}}^{2} e^{2}}{E_{\mathrm{gs}}} .
$$

Using (16) to determine the characteristic size $R_{\mathrm{gs}}$ of the perturber ion, we find

$$
a>\frac{6 Z_{\mathrm{i}}^{2}}{Z_{\mathrm{i}}+1} R_{\mathrm{gs}} \gtrsim \frac{6 Z_{\mathrm{i}}^{2}}{Z_{\mathrm{i}}+1}\left\langle r_{\mathrm{gs}}\right\rangle \geq 3\left\langle r_{\mathrm{gs}}\right\rangle .
$$

Thus, condition (17) is sufficient to validate the treatment of the perturber ions as pointlike.

Based on the results of the present work, we have developed an accurate approach to the determination of the occupation factors and effective statistical weights of the atomic and ionic states in plasmas. This approach and its results will be described in reference [11]. In particular, we will discuss there the effect of the correction term $\widetilde{Z}_{\mathrm{t}}(r)$ on the effective statistical weight values. Detailed calculations show that the effect of the nonzero-ion-size corrections on the effective statistical weight values for the excited states in dense nonideal plasmas, even for $\Gamma$ approaching unity, is rather small. The corrections of about $20 \%$ or smaller seem to be typical. The effect of the emitter ion polarization by the NN ion field on the spatial distributions of the neighbor ions is significantly weaker yet.

\section{Conclusion}

In plasmas, local microscopic conditions vary between individual ion locations. An accurate treatment of plasma density effects requires detailed knowledge of the probability distributions for these conditions. The traditional approach was to reduce the entire variety of local microscopic conditions to a single parameter: the Uniform Local Microfield strength at the location of the test ion. The ULM strength distribution is known quite accurately for a variety of macroscopic plasma conditions $[1,30]$, and has been used extensively (primarily in plasma diagnostics based on spectral line shapes [1]). However, as we have already pointed out, the ULM approximation can not be utilized to treat the problems where the electron wavefunctions with spatial range comparable to the interionic distances are considered, since on such scales the local microfield is 
not uniform. For the treatment of these problems, a more detailed description of the local microscopic conditions is required, and therefore the spatial distribution of the few nearest neighbor ions must be known.

In the present paper we have derived expression (3) for the distance-to-NN-ion distribution $P_{\mathrm{NN}}(r)$, expression (A.1) for the probability $P_{0}(r)$ to find no ions within $r$ from the test ion, and expressions $(7,6)$ for the distance-to-the-NNN-ion distribution $P_{\mathrm{NNN}}(\rho)$ and for the 3 -particle distribution $P_{\mathrm{NNN}}(r, \rho)$. We also present expression (11) for the distribution $P_{\text {rat }}(\zeta)$ of the ratio of the distances $\rho$ and $r$. For the ease of practical calculations involving these expressions, we have suggested simple analytical expressions $(5,10)$ for the potentials $U_{\mathrm{NN}}(r)$ and $U_{\mathrm{NNN}}(r, \rho)$. Comparison with classical-trajectory MD simulations showed that even with these semi-empirical potentials the accuracy of the theoretical predictions is high. We also study the distribution $P(\theta)$ of the angle between the directions to the $\mathrm{NN}$ and $\mathrm{NNN}$ ions.

The expression for $P_{\mathrm{NNN}}(r, \rho)$ here derived gives the simultaneous probability to find the $\mathrm{NN}$ ion at $r$ and the NNN ion at $\rho$. This quantity is especially useful for the treatment of non-resonant charge exchange, as it allows the effect of the perturbation produced by the nearby spectator ions on the charge exchange rates in plasma to be evaluated. For a given distance $r$ between the donor ("test ion") and the recipient ("NN ion") the distance $\rho$ to the spectator ("NNN ion") determines the mixing of the wavefunctions of the electron being removed. In the cases when the charge exchange occurs by tunneling, the rate of charge exchange depends strongly on the parabolic quantum numbers $n_{1}, n_{2}, m$ describing the initial state of the test ion optical electron in the NN ion field [19,31]. The field of the NNN ion, being not coaxial with the NN ion field, produces mixing of the states with different values of $n_{1}-n_{2}$ and $m$, and thus affects the tunneling rates from these states. Mixing coefficients may be rather easily found, since the effect of the NNN ion amounts to a spatial rotation of the states $\left|n_{1}, n_{2}, m\right\rangle$ by a finite angle $\alpha$ determined by $\zeta=\rho / r$ and $\theta$. Knowledge of the functions $P_{\mathrm{NNN}}(r, \rho)$ and $P(\theta)$ thus allows the magnitude of this effect to be estimated under various circumstances. This will be discussed in detail in reference [11].

The authors express their deepest gratitude to H.R. Griem and D. Salzmann for their help and concern on all stages of the present research.

\section{Appendix A: Accurate $P_{\mathrm{NN}}(r)$ derivation}

We choose the origin at the test ion location. In the conventional approach, the probability $P_{0}\left(r_{2}, r_{2}+\mathrm{d} r\right)$ for the absence of ions in the spherical shell $\left(r_{2}, r_{2}+\mathrm{d} r\right)$ around the test ion was considered independent on the "outcome of the trials" for the presence of an ion at any radius $r_{1}$ smaller than $r_{2}$. Consequently, the probability $P_{0}(r) \equiv P_{0}(0, r)$ for the absence of ions (besides the test one) in the sphere $r$ was written as the product of un- conditional probabilities for the absence of ions inside the thin spherical shells that constitute the sphere $r$ :

$$
P_{0}(r)=\lim _{\mathrm{d} r \rightarrow 0} \prod_{r^{\prime}=0, \mathrm{~d} r, \ldots, r-\mathrm{d} r} P_{0}\left(r^{\prime}, r^{\prime}+\mathrm{d} r\right) .
$$

In reality, however, $P_{0}\left(r_{2}, r_{2}+\mathrm{d} r\right)$ depends on the presence or absence of ions at all $r_{1}<r_{2}$. Therefore, the probability $P_{0}(r)$ must be written as the product of conditional probabilities for the absence of ions inside the spherical shells $\left(r^{\prime}, r^{\prime}+\mathrm{d} r\right)$ provided there are no ions inside $r^{\prime}$, i.e.,

$P_{0}(r)=\lim _{\mathrm{d} r \rightarrow 0} \prod_{r^{\prime}=0, \mathrm{~d} r, \ldots, r-\mathrm{d} r} P_{0}\left(r^{\prime}, r^{\prime}+\mathrm{d} r \mid\right.$ no ions inside $\left.r^{\prime}\right)$.

The first factor in this product is explicitly equal to

$$
P_{0}(0, \mathrm{~d} r \mid \text { no neighbor ions at origin }) \equiv P_{0}(0, \mathrm{~d} r),
$$

since the probability for the absence of ions (besides the test one) at the origin is unity.

For $r^{\prime}>0$, in the absence of ions inside $r^{\prime}$, the potential at $r^{\prime}$ is given by $U_{\mathrm{NN}}\left(r^{\prime}\right)$, and the density of ions at $r^{\prime}$ is given by $n_{\mathrm{i}} \exp \left(-\beta Z_{\mathrm{i}} e U_{\mathrm{NN}}\left(r^{\prime}\right)\right)$. This enables us to express:

$$
\begin{aligned}
& P_{0}\left(r^{\prime}, r^{\prime}+\mathrm{d} r \mid \text { no ions inside } r^{\prime}\right)= \\
& \lim _{V \rightarrow \infty ;}{ }_{V}^{N=n_{\mathrm{i}}=\text { const. }} \\
& =\lim _{N \rightarrow \infty}\left[1-\frac{4 \pi r^{\prime 2} \mathrm{~d} r^{\prime} \exp \left\{-\beta Z_{\mathrm{i}} e U_{\mathrm{NN}}\left(r^{\prime}\right)\right\}}{V-\frac{4}{3} \pi r^{\prime 3}}\right]^{N} \\
& =\exp \left[-4 \pi \bar{n}_{\mathrm{i}} r^{\prime 2} \mathrm{~d} r^{\prime} \exp \left\{-\beta Z_{\mathrm{i}} e U_{\mathrm{NN}} r^{\prime 2} \mathrm{~d} r^{\prime} \exp \left\{-\beta Z_{\mathrm{i}} e U_{\mathrm{NN}}\left(r^{\prime}\right)\right\}\right] .\right.
\end{aligned}
$$

The product can then be readily evaluated:

$$
\begin{aligned}
& P_{0}(r)= \\
& \lim _{\mathrm{d} r \rightarrow 0} \prod_{r^{\prime}=0, \mathrm{~d} r, \ldots, r-\mathrm{d} r} \exp \left[-4 \pi \bar{n}_{\mathrm{i}} r^{\prime 2} \mathrm{~d} r^{\prime} \exp \left\{-\beta Z_{\mathrm{i}} e U_{\mathrm{NN}}\left(r^{\prime}\right)\right\}\right] \\
& \quad=\exp \left[-4 \pi \bar{n}_{\mathrm{i}} \int_{0}^{r} r^{\prime 2} \mathrm{~d} r^{\prime} \exp \left\{-\beta Z_{\mathrm{i}} e U_{\mathrm{NN}}\left(r^{\prime}\right)\right\}\right] .
\end{aligned}
$$

The NN distribution function $P_{\mathrm{NN}}(r) \mathrm{d} r$ is given by the decrease of $P_{0}(r)$ across $\mathrm{d} r$, i.e.

$$
P_{\mathrm{NN}}(r) \equiv-\frac{\mathrm{d} P_{0}(r)}{\mathrm{d} r}
$$

yielding the expression (3).

A simpler version of the same derivation is as follows. The probability $P_{0}(r+\mathrm{d} r)$ for the absence of ions in the sphere of radius $r+\mathrm{d} r$ around the test ion is given by the product of probabilities

$$
P_{0}(r) P_{0}(r, r+\mathrm{d} r \mid \text { no ions inside } r) .
$$


In the absence of ions (besides the test one) in the sphere $r$, the ion density in the spherical shell $(r, r+\mathrm{d} r)$ is given by $\bar{n}_{\mathrm{i}} \exp \left\{-\beta Z_{\mathrm{i}} e U_{\mathrm{NN}}(r)\right\}$. Thus,

$P_{0}(r, r+\mathrm{d} r \mid$ no ions inside $r) \equiv$

$$
1-\bar{n}_{\mathrm{i}} \exp \left\{-\beta Z_{\mathrm{i}} e U_{\mathrm{NN}}(r)\right\} 4 \pi r^{2} \mathrm{~d} r
$$

and

$$
P_{0}(r+\mathrm{d} r)=P_{0}(r)\left[1-\bar{n}_{\mathrm{i}} \exp \left\{-\beta Z_{\mathrm{i}} e U_{\mathrm{NN}}(r)\right\} 4 \pi r^{2} \mathrm{~d} r\right],
$$

which implies

$$
\frac{\mathrm{d} P_{0}(r)}{\mathrm{d} r}=-\bar{n}_{\mathrm{i}} \exp \left\{-\beta Z_{\mathrm{i}} e U_{\mathrm{NN}}(r)\right\} 4 \pi r^{2} P_{0}(r) .
$$

Given the boundary condition $P_{0}(0) \equiv 1$, the expressions (3, A.1) readily follow. It is noteworthy that this result is a manifestation of the general fact that whenever the particle density immediately outside the sphere of radius $r$ is given by a function $n(r)$ integrable between 0 and $r$, the probability for the absence of particles inside $r$ is given by

$$
P_{0}(r)=\exp \left\{-4 \pi \int_{0}^{r} n\left(r^{\prime}\right) r^{\prime 2} \mathrm{~d} r^{\prime}\right\},
$$

regardless of the conditions inside the sphere. For $n(r)$ given by the Boltzmann formula

$$
n(r)=\bar{n} \exp \{-\beta u(r)\}
$$

where $u(r)$ is the potential energy of a particle at $r$, the probability $P_{0}(r)$ is always given by

$$
P_{0}(r)=\exp \left\{-4 \pi \bar{n} \int_{0}^{r} \exp \left\{-\beta u\left(r^{\prime}\right)\right\} r^{\prime 2} \mathrm{~d} r^{\prime}\right\},
$$

and thus

$$
\begin{aligned}
& P_{\mathrm{NN}}(r)= \\
& 4 \pi \bar{n} r^{2} \exp \left\{-\beta u(r)-4 \pi \bar{n} \int_{0}^{r} \exp \left\{-\beta u\left(r^{\prime}\right)\right\} r^{\prime 2} \mathrm{~d} r^{\prime}\right\} .
\end{aligned}
$$

This form is, therefore, general; the physics of the interactions involved finds its manifestation in $u(r)$ only.

\section{References}

1. H.R. Griem, Principles of Plasma Spectroscopy (Cambridge Univ. Press, 1997).

2. Or at least of its low-frequency part. Treatment of the free electron collision effects on atomic states (spectral line broadening by elastic and inelastic collisions, and transitions caused by inelastic collisions) is beyond the scope of the present work; see [1] for a comprehensive discussion of this subject.

3. P. Malnoult, B. d'Etat, Hoe Nguyen, Phys. Rev. A 40, 1983 (1989).

4. D. Salzmann, J. Stein, I.B. Goldberg, R.H. Pratt, Phys. Rev. A 44, 1270 (1991).

5. E. Leboucher-Dalimier, A. Poquérusse, P. Angelo, Phys. Rev. E 47, R1467 (1993).

6. R.K. Janev, L.P. Presnyakov, V.P. Shevelko, Physics of Highly Charged Ions (Springer-Verlag, Berlin, 1985).

7. G.B. Zimmerman, R.M. More, J. Quant. Spectrosc. Radiat. Transfer 23, 517 (1980).

8. V.E. Fortov, I.T. Yakubov, Physics of Nonideal Plasma (Hemisphere Publ., New York, 1990); in Russian: Fizika neideal'noi plazmy (IVTAN, 1984).

9. D.G. Hummer, D. Mihalas, Astrophys. J. 331, 794 (1988).

10. D. Salzmann, Atomic Physics in Hot Plasmas (Oxford Univ. Press, New York, 1998).

11. D.V. Fisher, Y. Maron, Eur. Phys. J. D (to be submitted).

12. The Opacity Project, edited by M.J. Seaton (IOP publ., Bristol, 1995), Vol. 1.

13. C.A. Iglesias, F.J. Rogers, Astrophys. J. 443, 460 (1995).

14. D.P. Kilcrease, R.C. Mancini, C.F. Hooper Jr, Phys. Rev. E 48, 3901 (1993).

15. M.S. Murillo, J.C. Weisheit, Phys. Rep. 302, 1 (1998).

16. P. Sauvan, E. Leboucher-Dalimier, P. Angelo, H. Derfoul, T. Ceccotti, A. Poquérusse, A. Calisti, B. Talin, J. Quant. Spectrosc. Radiat. Transfer 65, 511 (2000).

17. J. Stein, I.B. Goldberg, D. Shalitin, D. Salzmann, Phys. Rev. A 39, 2078 (1989).

18. J. Stein, D. Salzmann, Phys. Rev. A 45, 3943 (1992).

19. T.P. Grodzanov, R.K. Janev, Phys. Rev. A 17, 880 (1978).

20. A. Unsöld, Z. Astrophys. 24, 355 (1948).

21. I.Z. Fisher, Statistical Theory of Liquids (Chicago Univ. Press, 1964); in Russian: Statisticheskaia Teoriia Zhidkostei (Fizmatgiz, Moscow, 1961).

22. J.P. Hansen, I.R. McDonald, Theory of Simple Liquids (Acad. Press, London, 1976).

23. L.D. Landau, E.M. Lifshitz, v.5 Statistical Physics: Part 1, 3rd edn. (Butterworth, 1980).

24. J.F. Springer, M.A. Pokrant, F.A. Stevens Jr, J. Chem. Phys 58, 4863 (1973).

25. M.W.C. Dharma-Wardana, F. Perrot, Phys. Rev. A 26, 2096 (1982).

26. F.J. Rogers, Phys. Rev. A 29, 868 (1984).

27. R. Cauble, M. Blaha, J. Davis, Phys. Rev. A 29, 3280 (1984).

28. C. Deutsch, Phys. Lett. A 60, 317 (1977)

29. Ya.B. Zel'dovich, Yu.P. Raizer, Physics of Shock Waves and High-Temperature Hydrodynamic Phenomena (Acad. Press, New York, 1966).

30. J.W. Dufty, D.B. Boercker, C.A. Iglesias, Phys. Rev. A 31, 1681 (1985).

31. D.V. Fisher, Y. Maron, L.P. Pitaevskii, Phys. Rev. A 58, 2214 (1998). 